\section{A atenção primária em regiões de saúde: política, estrutura e organização}

\author{
Primary care in health regions: policy, structure, \\ and organization
}

\section{La atención primaria en regiones de salud: política, estructura y organización}

\section{Resumo}

A atenção primária à saúde (APS) concebida como coordenadora do cuidado e ordenadora da rede regionalizada de atenção à saúde (RAS) é um dos condicionantes da dinâmica regional da saúde. O objetivo do artigo é identificar as dimensões política, de estrutura e de organização da APS em diferentes regiões do Brasil; assume-se que estas dimensões podem explicar, se não o todo, pelo menos parte importante do funcionamento da APS em cenário regional. Foram realizadas 84 entrevistas com atores-chave em cinco regiões de saúde. Essas regiões foram selecionadas com base na diversidade de situações socioeconômicas, territoriais e de organização do sistema de saúde. Apesar da heterogeneidade das RAS, notam-se traços comuns. Na dimensão da política, observou-se fragilidade na cooperação intergovernamental e no protagonismo da esfera estadual, além da incapacidade da Comissão Intergestores Bipartite em se configurar como espaço de planejamento e pactuação. $\mathrm{Na}$ dimensão estrutura ficou clara a insuficiência de condições que assegurem minimamente a execução de funções essenciais da APS. Pontos críticos são escassez, má distribuição e problemas de qualificação de recursos humanos, além do subfinanciamento das ações. $\mathrm{Na}$ dimensão organização são visíveis as dificuldades para se romper a fragmentação dos serviços. A APS não consegue assumir seu papel de coordenadora do cuidado, e observa-se a ausência de um modus operandi capaz de atender às necessidades dos usuários considerando as especificidades de cada região. A superação dos constrangimentos identificados é central para o fortalecimento do próprio SUS como sistema público, universal, equânime e integral.

Atenção Primária à Saúde; Regionalização; Sistemas de Saúde
Aylene Bousquat 1

Lígia Giovanella 2

Marcia Cristina Rodrigues Fausto 1

Maria Guadalupe Medina 3

Cleide Lavieri Martins 1

Patty Fidelis Almeida 4

Estela Marcia Saraiva Campos 5

Paulo Henrique dos Santos Mota 1

doi: 10.1590/0102-311X00099118

\section{Correspondência}

P. H. S. Mota

Faculdade de Saúde Pública, Universidade de São Paulo. Av. Dr. Arnaldo 715, São Paulo, SP 01246-904, Brasil. paulohsmota@gmail.com

1 Faculdade de Saúde Pública, Universidade de São Paulo, São Paulo, Brasil.

2 Escola Nacional de Saúde Pública Sergio Arouca, Fundação Oswaldo Cruz, Rio de Janeiro, Brasil.

3 Instituto de Saúde Coletiva, Universidade Federal da Bahia, Salvador, Brasil.

4 Instituto de Saúde Coletiva, Universidade Federal Fluminense, Niterói, Brasil.

5 Universidade Federal de Juiz de Fora, Juiz de Fora, Brasil. 


\section{Introdução}

A regionalização, diretriz e decisivo componente organizativo do Sistema Único de Saúde (SUS), vem impulsionando, na fase atual da política de saúde brasileira, a constituição de redes de atenção à saúde integradas e regionalizadas. No entanto, esse é um processo em curso e ainda incipiente. A experiência internacional indica que, em países com sistemas universais, a estratégia de redes regionalizadas e hierarquizadas tem sido o principal meio para a garantia de cobertura e acesso equânime aos serviços e ações de saúde. Três pressupostos constituem a base para a operacionalização dessa estratégia: suficiência dos serviços de saúde de acordo com as necessidades da população, responsabilização pela atenção aos usuários, e coordenação assistencial 1 .

A região de saúde deve representar a base territorial e populacional para a operação da rede regionalizada de atenção à saúde (RAS), com definição de perfil assistencial, considerando as necessidades de saúde da população. Não se trata de um território criado burocraticamente pelo sistema de saúde, mas o reconhecimento de um espaço onde se estabelecem as relações econômicas, sociais, políticas e culturais e, também, os cuidados em saúde 2. Nesse sentido, as RAS demandam uma renovada região de saúde, que construiria, por meio das reconfigurações das fronteiras, um território sanitário comum, tendo como objetivo a garantia do cuidado integral em saúde 3 . A oferta de serviços de saúde nesse espaço regional está condicionada à capacidade de pactuação entre gestores (municipais, estaduais e federal), num cenário marcado por condições desiguais de negociação e motivada por interesses diversos 4,5. Inúmeras são as dificuldades para a consolidação desse espaço regional como pilar para estruturação e organização dos serviços de saúde no país. Ressalta-se que a política de saúde brasileira tem traços que não facilitam a conformação de redes regionalizadas. Ao contrário, tem favorecido a conformação de sistemas municipais de saúde atomizados e desarticulados e com baixa cooperação intergovernamental 4,6,7,8,9.

Vale ressaltar que o conceito de redes regionalizadas, aqui utilizado, é aquele que se desenha com base na concepção da saúde como um direito, e tem como objetivo contribuir para que a integralidade seja alcançada. Essa lógica certamente não é a mesma que pode ser observada nas propostas de redes assistenciais integradas implantadas sob a égide do mercado, focada na integração de prestadores de serviços de saúde 1 .

$\mathrm{Na}$ conformação da RAS em sistemas universais de saúde está imbricada a presença de uma atenção primária à saúde (APS) integral e resolutiva. Para a efetivação da RAS, a APS deve assumir seu papel como porta de entrada preferencial ao sistema, aberta e resolutiva, garantindo o acesso às ações de saúde, em suma, deve ordenar o sistema. Simultaneamente, para a APS a regionalização é também crucial, posto que a qualificação de suas intervenções demanda a integração com os demais níveis assistenciais, quase nunca disponibilizados em sua totalidade nos limites dos territórios municipais 3,10. Só a articulação da APS com os demais componentes da RAS é que pode garantir as condições para que este nível de atenção possa exercer os atributos de cuidado regular e contínuo, em prol da integralidade das ações e de impactos positivos na saúde da população 11,12.

Nessa direção, assume-se que a APS é um dos componentes centrais para que se avalie se a implantação da RAS caminha efetivamente na garantia da integralidade ou não. Dessa forma, o objetivo deste artigo é analisar se a APS assume seu papel como ordenadora do sistema de saúde em diferentes cenários regionais brasileiros.

\section{Metodologia}

A presente investigação é parte da pesquisa Política, Planejamento e Gestão das Regiões e Redes de Atenção à Saúde no Brasil. Partiu-se da premissa que a APS, como nível de atenção coordenador do cuidado e ordenador da RAS, seria um dos condicionantes da dinâmica regional da saúde. Nessa perspectiva, optou-se por uma análise multidimensional, na qual se agregam três dimensões que podem explicar, se não o todo, pelo menos parte importante do funcionamento da APS nos sistemas regionalizados: as dimensões política, estrutura e organização 13 .

A primeira dimensão se refere ao processo político propriamente dito, e procura identificar os espaços de atuação, negociação e conflito na região, os processos e fluxos decisórios e a condução da política e as funções exercidas por cada instituição nas decisões sobre a APS. A estrutura é traduzida 
na disponibilidade e suficiência de recursos humanos, físicos, financeiros e as formas de contratação de recursos humanos, serviços e exames complementares presentes na política de regionalização. A dimensão organização, por sua vez, pretende identificar o planejamento, a gestão, os instrumentos de monitoramento e avaliação, além da integração sistêmica entre serviços e a coordenação do cuidado pela APS.

Baseado nesse quadro analítico foram realizados estudos em cinco regiões de saúde, selecionadas intencionalmente, de forma a expressar a diversidade de situações socioeconômicas, territoriais e de organização do sistema de saúde. Para tanto, partiu-se de três critérios: territorial-administrativo, desenvolvimento socioeconômico, e oferta e complexidade do sistema de saúde. Foram então escolhidas cinco regiões de saúde das cinco macrorregiões brasileiras - Manaus, Entorno e Alto do Rio Negro (Amazonas), no Norte; Baixada Cuiabana (Mato Grosso), no Centro-oeste; Petrolina (Pernambuco) e Juazeiro (Bahia), no Nordeste; Norte-Barretos e Sul-Barretos (São Paulo), no Sudeste; e Carbonífera e Costa Doce (Rio Grande do Sul), no Sul, aqui denominadas, respectivamente, de Manaus, Cuiabana, PEBA, Barretos e Carbonífera. Ressalta-se que Petrolina e Juazeiro foram consideradas como uma única região pela história de constituição da importante rede interestadual denominada PEBA (Rede Interestadual de Atenção à Saúde do Vale Médio do São Francisco), assim como Barretos aglutinou as regiões de Norte-Barretos e Sul-Barretos, pois foi esta a dinâmica regional observada no campo de pesquisa ${ }^{14}$. O campo foi realizado entre agosto de 2015 e junho de 2016. Detalhes sobre a seleção e caracterização das regiões foram apresentados por Viana et al. 14.

Em cada região foram incluídos três municípios para a realização do campo, sendo um obrigatoriamente o município polo. Os demais foram escolhidos, de forma intencional, com base na oferta de estabelecimentos de saúde, porte populacional, distância do município polo e preponderância na articulação da política regional 14 .

Os resultados aqui apresentados são provenientes da análise dos questionários estruturados aplicados a atores-chave selecionados nas cinco regiões. Esse questionário incluiu questões relativas à APS nas três dimensões destacadas anteriormente, incluindo diversos formatos de questões (sim/ não; escala Likert; semiabertas). As respostas foram analisadas em porcentuais no caso das perguntas sim/não, e as questões respondidas na escala Likert foram transformadas em escores (variando de sempre $=5$ até nunca $=1$ ), sendo apresentados em escores médios 14 .

Os atores-chave selecionados tinham experiência em APS na região e eram gestores e/ou gerentes de unidades básicas de saúde (UBS). Os gestores eram secretários municipais de saúde, coordenadores municipais de APS, diretores regionais, coordenadores regionais de APS ou função correlata. Para a entrevista com os gerentes de UBS, em cada município foram escolhidas, por amostra intencional, UBS que representassem a diversidade da qualidade da estrutura destes serviços em base a uma tipologia das UBS brasileiras, construídas valendo-se dos resultados do censo brasileiro de infraestrutura das UBS realizado no processo de avaliação externa do ciclo 1 do PMAQ-AB (Programa Nacional de Melhoria do Acesso e da Qualidade da Atenção Básica) 15.

No Quadro 1 são elencadas as variáveis, por dimensão, analisadas neste artigo.

Um dos limites deste estudo reside na influência da inserção profissional e regional do entrevistado nas respostas obtidas, já que é muito pouco provável que um entrevistado consiga referenciar todo o panorama das dimensões política, de estrutura e de organização da APS nas regiões. Esse limite foi minimizado pela inclusão de entrevistados com inserções profissionais distintas e vinculados a diferentes municípios da região.

O estudo foi aprovado pelo Comitê de Ética da Faculdade de Medicina da Universidade de São Paulo, com o número de processo 071/15, CAAE: 42787815.9.1001.0065, de acordo com a norma do Conselho Nacional de Saúde Resolução no 466/2012.

\section{Resultados e discussão}

Foram realizadas 84 entrevistas em 21 municípios das cinco regiões. A maioria dos respondentes foi do sexo feminino (76,2 \%), com idade média de 38,5 anos. Os enfermeiros responderam pelo maior número de entrevistas em todas as regiões. A distribuição percentual de gestores e gerentes de UBS foram semelhantes nas cinco regiões de estudo (Tabela 1). 


\section{Quadro 1}

Dimensões e variáveis de análise.

\begin{tabular}{|c|c|}
\hline Dimensões & Variáveis \\
\hline Política & $\begin{array}{l}\text { - Instituição organizadora da APS } \\
\text { - Conflitos na tomada de decisão na APS } \\
\text { - Instâncias/órgãos importantes para tomada de decisão em APS } \\
\text { - Papel da CIR na organização da APS } \\
\text { - Temas discutidos na CIR relacionados à APS }\end{array}$ \\
\hline Estrutura & $\begin{array}{l}\text { - Suficiência da cobertura da APS } \\
\text { - Suficiência na oferta de consultas especializadas e SADT de média complexidade } \\
\text { - Existência de grupos populacionais sem cobertura de APS } \\
\text { - Critérios de risco e vulnerabilidade para definição de população adscrita } \\
\text { - Suficiência de recursos humanos na APS } \\
\text { - Natureza jurídica e vínculo na contratação de recursos humanos } \\
\text { - Participação relativa das esferas federativas no financiamento } \\
\text { - Investimento para APS }\end{array}$ \\
\hline Organização & $\begin{array}{l}\text { - Instrumentos de planejamento da APS } \\
\text { - Avaliação da APS } \\
\text { - Porta de entrada preferencial na RAS } \\
\text { - Resolutividade da APS } \\
\text { - Integração entre os níveis assistenciais } \\
\text { - Garantia de atenção oportuna } \\
\text { - Coordenação do cuidado pela APS } \\
\text { - Conhecimento dos tempos de espera para atenção especializada e procedimentos }\end{array}$ \\
\hline
\end{tabular}

APS: atenção primária à saúde; CIR: Comissão Intergestores Regional; SADT: Serviços de Apoio

Diagnóstico e Terapêutico.

Tabela 1

Perfil dos entrevistados. Regiões estudadas, 2015/2016.

\begin{tabular}{|c|c|c|c|c|c|c|c|c|c|c|}
\hline & \multicolumn{2}{|c|}{ Barretos } & \multicolumn{2}{|c|}{ Manaus } & \multicolumn{2}{|c|}{ Cuiabana } & \multicolumn{2}{|c|}{ PEBA } & \multicolumn{2}{|c|}{ Carbonífera } \\
\hline & $\mathbf{n}$ & $\%$ & $\mathrm{n}$ & $\%$ & $\mathbf{n}$ & $\%$ & $\mathbf{n}$ & $\%$ & $\mathbf{n}$ & $\%$ \\
\hline \multicolumn{11}{|l|}{ Sexo } \\
\hline Masculino & 3 & 17,6 & 4 & 33,3 & 3 & 18,8 & 6 & 25,0 & 4 & 26,7 \\
\hline Feminino & 14 & 82,4 & 8 & 66,7 & 13 & 81,2 & 18 & 75,0 & 11 & 73,3 \\
\hline \multicolumn{11}{|l|}{ Cargo } \\
\hline Gestor & 6 & 35,3 & 4 & 33,3 & 5 & 31,3 & 9 & 37,5 & 5 & 33,3 \\
\hline Gerente de UBS & 11 & 64,7 & 8 & 66,7 & 11 & 68,7 & 15 & 62,5 & 10 & 66,7 \\
\hline \multicolumn{11}{|l|}{ Formação } \\
\hline Enfermagem & 12 & 70,5 & 8 & 66,6 & 10 & 62,5 & 18 & 75,0 & 7 & 46,6 \\
\hline Outros saúde * & 2 & 11,8 & 0 & 0,0 & 2 & 12,5 & 1 & 4,2 & 3 & 20,0 \\
\hline Medicina & 1 & 5,9 & 2 & 16,7 & 1 & 6,3 & 1 & 4,2 & 0 & 0,0 \\
\hline Outros ** & 2 & 11,8 & 2 & 16,7 & 3 & 18,7 & 4 & 16,6 & 5 & 33,4 \\
\hline $\begin{array}{l}\text { Total de } \\
\text { entrevistados }\end{array}$ & \multicolumn{2}{|c|}{17} & \multicolumn{2}{|c|}{12} & \multicolumn{2}{|c|}{16} & \multicolumn{2}{|c|}{24} & \multicolumn{2}{|c|}{15} \\
\hline
\end{tabular}

PEBA: Rede Interestadual de Atenção à Saúde do Vale Médio do São Francisco (Petrolina, Pernambuco e Juazeiro, Bahia); UBS: unidades básicas de saúde.

* Serviço Social, Fisioterapia, Odontologia, Psicologia.

** Administração, Direito, Ciências Matemáticas, Pedagogia e Ensino Médio completo. 


\section{Dimensão política}

As Secretarias Municipais de Saúde (SMS) são citadas como as principais organizadoras da APS em todos os casos. Nas regiões Cuiabana e Manaus esta característica é mais evidente, sendo que as SMS foram citadas por 73,3\% e 91,7\% dos entrevistados, respectivamente. A estrutura regional das Secretarias Estaduais de Saúde (SES) aparece com mais peso em Barretos (41,1\%) e PEBA (31,5\%) (Tabela 2).

Os conflitos mais importantes na tomada de decisão sobre a APS, na fala dos gestores, são aqueles entre as instâncias regionais e os municípios nas regiões de PEBA, Barretos e Cuiabana. Por outro lado, nas regiões de Manaus e Carbonífera o conflito assume uma feição intermunicipal (Tabela 2). Esse dado vai ao encontro dos resultados de Viana et al. 13 que concluíram que, nessas cinco regiões, os processos de regionalização mais consolidados foram evidenciados na região de PEBA, seguida pelas regiões de Barretos e Cuiabana.

Chama a atenção que os conflitos entre os níveis federal e municipal assumam menor importância relativa, com exceção da Baixada Cuiabana. Esse perfil pode ser interpretado pela preponderância do Ministério da Saúde na condução política da expansão da APS no Brasil, sustentada, em muitos casos, pelos incentivos financeiros do governo federal. Esse protagonismo é tão evidente que não há sequer espaço para a disputa política 12 . As disputas entre estado e União são percebidas diferentemente nas regiões, sendo mais referidas naquelas com processos mais consolidados de regionalização, com exceção da região de Barretos.

É interessante citar que os conflitos para a tomada de decisão sobre a APS, do ponto de vista dos gestores, envolvem principalmente os municípios e a instância regional e gestores públicos e profissionais de saúde. Analisando apenas os gerentes das UBS, que vivenciam diariamente o contato com os usuários e com menor contato com os demais municípios, o conflito se dá entre médicos especialistas e outros profissionais (média de 64,3\%); entre gestores públicos e profissionais de saúde (média de 60,7\%); e entre médicos do Programa Mais Médicos e demais profissionais (57,1\%).

No tocante aos órgãos e instâncias que mais influenciam a tomada de decisão sobre APS na região, observou-se consenso entre os entrevistados nas cinco regiões. Em primeiro lugar está o Ministério da Saúde, seguido pelas SMS da região e pelas estruturas regionais e o nível central das SES. Unanimidade também foi observada quanto à importância dos espaços para a tomada de decisão. Em todas as regiões foram pontuadas em primeiro lugar a Comissão Intergestores Regional (CIR) e Comissão Intergestores Bipartite (CIB), seguidas pelo Conselho dos Secretários Municipais de Saúde (COSEMS), Comissão Intergestores Tripartite e Conselhos de Saúde. O Ministério Público, mídia e estabelecimentos de média e alta complexidade, públicos e privados contratados, pelo SUS foram indicados como tendo influência média na tomada de decisão sobre APS na região, com variação entre elas (Tabela 2).

A CIR foi apontada pela maioria dos gestores das regiões de Barretos, PEBA e Carbonífera como a instância que discute e define os fluxos assistenciais, especialmente entre a APS e a Atenção Especializada. Ao passo que para as regiões de Manaus e Cuiabana, ainda com processo de regionalização incipiente, esses fluxos não são estabelecidos na CIR. Esse ponto é ainda mais expressivo quando se reconhece que, para a integralidade da atenção, os serviços de APS necessitam da garantia do acesso a exames complementares e especialistas em tempo oportuno. Análises da CIR em outros cenários 3 ratificam as avaliações positivas por parte de gestores do SUS em relação ao seu potencial como espaço de governança regional, sobretudo para os temas relacionados ao acesso e definição de fluxos para a atenção especializada e hospitalar. Vale ressaltar que outros estudos indicam que, mesmo na CIR, a discussão do fluxo da APS para os demais níveis tem seu foco na premência em garantir retaguarda terapêutica, e não em questões relacionadas ao próprio fortalecimento da APS, tema ainda marginal e circunscrito aos limites municipais 3 .

O financiamento é um dos temas mais frequentes relacionado à APS discutidos na CIR, assim como a cobertura de serviços, seguido da articulação da APS com as redes de atenção, com exceção de Manaus e Cuiabana. A preponderância do tema do financiamento reflete o modelo de indução financeira para a construção das RAS, que tem fomentado o planejamento e organização da rede centrada no município, além de competição entre os mesmos para dispor de fundos federais $4,6,7$.

Por outro lado, os gerentes de UBS demonstram alto grau de desconhecimento do que se discute na CIR, com metade deles não sendo capaz de responder à questão. Um estudo realizado em regiões 


\section{Tabela 2}

Percepção dos entrevistados sobre espaços de atuação, decisão e conflito acerca da atenção primária à saúde (APS). Regiões estudadas, $2015 / 2016$.

\begin{tabular}{|c|c|c|c|c|c|}
\hline Espaços & $\begin{array}{l}\text { Barretos } \\
\text { Sim }(\%)\end{array}$ & $\begin{array}{l}\text { Manaus } \\
\text { Sim (\%) }\end{array}$ & $\begin{array}{l}\text { Cuiabana } \\
\text { Sim (\%) }\end{array}$ & $\begin{array}{c}\text { PEBA } \\
\text { Sim (\%) }\end{array}$ & $\begin{array}{l}\text { Carbonífera } \\
\text { Sim (\%) }\end{array}$ \\
\hline \multicolumn{6}{|l|}{ Principal instância organizadora da APS (\%) } \\
\hline SMS & 58,9 & 91,7 & 73,3 & 57,8 & 50 \\
\hline Estrutura regional da SES & 41,1 & 0 & 13,3 & 31,5 & 28,5 \\
\hline \multirow[t]{2}{*}{ SES (nível central) } & 0 & 8,3 & 13,4 & 10,7 & 21,5 \\
\hline & $\operatorname{Sim}(\%)$ & $\operatorname{Sim}(\%)$ & $\operatorname{Sim}(\%)$ & $\operatorname{Sim}(\%)$ & $\operatorname{Sim}(\%)$ \\
\hline \multicolumn{6}{|l|}{ Atores envolvidos em conflitos na tomada de decisão sobre a APS } \\
\hline Municípios - Governo estadual & 66,7 & 50,0 & 68,7 & 63,1 & 53,3 \\
\hline Gestor público e profissionais de saúde & 64,3 & 33,3 & 62,5 & 52,6 & 73,3 \\
\hline Médicos especialistas - outros profissionais & 64,3 & 41,7 & 62,5 & 57,9 & 66,7 \\
\hline Municípios - instância regional & 46,7 & 50,0 & 68,7 & 47,4 & 73,3 \\
\hline Município - Governo federal & 28,6 & 42,7 & 73,3 & 47,4 & 60,0 \\
\hline Governo estadual - Governo federal & 28,6 & 18,2 & 60,0 & 52,6 & 40,0 \\
\hline \multirow[t]{2}{*}{ Gestor público - prestador privado lucrativo } & 28,6 & 18,2 & 31,2 & 26,3 & 33,3 \\
\hline & Escore & Escore & Escore & Escore & Escore \\
\hline \multicolumn{6}{|l|}{ Influência de órgãos na tomada de decisão sobre a APS * } \\
\hline Ministério da Saúde & 4,1 & 3,6 & 4,3 & 4,4 & 4,1 \\
\hline Secretarias municipais de saúde da região de saúde & 4,0 & 3,6 & 3,8 & 4,1 & 4,1 \\
\hline Estrutura regional da SES & 3,7 & 3,0 & 3,7 & 4,0 & 4,0 \\
\hline SES (nível central) & 3,7 & 2,7 & 3,7 & 3,9 & 3,6 \\
\hline Ministério Público & 3,2 & 2,1 & 3,3 & 3,1 & 2,5 \\
\hline \multirow[t]{2}{*}{ Órgãos do sistema judiciário } & 2,8 & 1,9 & 2,1 & 2,7 & 2,2 \\
\hline & Escore & Escore & Escore & Escore & Escore \\
\hline \multicolumn{6}{|l|}{ Influência de espaços políticos para a tomada de decisão sobre a APS * } \\
\hline Comissão Intergestores Regional & 4,2 & 3,6 & 3,4 & 4,3 & 3,6 \\
\hline Comissão Intergestores Bipartite & 4,1 & 3,5 & 3,4 & 4,2 & 3,6 \\
\hline COSEMS & 3,9 & 3,1 & 3,3 & 4,0 & 3,5 \\
\hline Comissão Intergestores Tripartite & 3,7 & 3,0 & 3,3 & 3,9 & 3,3 \\
\hline \multirow[t]{2}{*}{ Conselhos de saúde } & 3,6 & 2,7 & 3,0 & 3,9 & 3,3 \\
\hline & Escore & Escore & Escore & Escore & Escore \\
\hline \multicolumn{6}{|l|}{ Influência de prestadores na tomada de decisão sobre a APS * } \\
\hline Estabelecimentos públicos de média e alta complexidade & 3,0 & 2,6 & 2,7 & 3,2 & 3,0 \\
\hline Estabelecimentos privados lucrativos contratados/conveniados ao SUS & 3,0 & 2,2 & 2,4 & 2,9 & 2,8 \\
\hline $\begin{array}{l}\text { Estabelecimentos privados não lucrativos contratados/conveniados ao SUS } \\
\text { de média e alta complexidade }\end{array}$ & 2,5 & 1,9 & 2,4 & 2,7 & 2,7 \\
\hline
\end{tabular}

COSEMS: Conselho dos Secretários Municipais de Saúde; PEBA: Rede Interestadual de Atenção à Saúde do Vale Médio do São Francisco (Petrolina, Pernambuco e Juazeiro, Bahia); SES: Secretaria Estadual de Saúde; SMS: Secretaria Municipal de Saúde; SUS: Sistema Único de Saúde.

* Escores variando de muito alta $=5$ até muito baixa $=1$. 
da Bahia 3 também demonstrou desconhecimento dos gestores municipais em relação à dinâmica e situação da APS em contexto regional.

Os achados apresentados indicam a necessidade de um maior protagonismo político da CIR. Ainda que a APS seja de execução municipal, a CIR deveria representar um espaço de planejamento e pactuação da qualidade da oferta dos serviços, de otimização e racionalização da utilização dos recursos especializados.

Um novo fôlego no fortalecimento das ações regionais e na construção das RAS pode resultar da implantação da Resolução no 37/2018 da Comissão Intergestores Tripartite, que visa à instituição do espaço regional ampliado como referência para a alocação de recursos financeiros pelos entes federados, mas ainda não há dados empíricos para se confirmar esta potencialidade. Vale lembrar que isso cobra uma mudança profunda no federalismo brasileiro, marcado desde suas origens por assimetria de poder político e econômico e relações pouco cooperativas entre os níveis de governo 16 .

\section{Dimensão estrutura}

Quando indagados sobre a suficiência dos serviços de APS, atenção especializada e Serviços de Apoio Diagnóstico e Terapêutico (SADT) de média complexidade, os entrevistados foram críticos em suas respostas considerando em geral a oferta insuficiente (Figura 1). A região de Barretos foi a única em que pelo menos $40 \%$ dos entrevistados consideraram a oferta suficiente, o que deve ser analisado criticamente, pois o percentual ainda pode ser considerado insatisfatório. A cobertura de serviços de APS foi a melhor avaliada nas cinco regiões, apesar da grande variação percentual. Mesmo nas regiões de PEBA e Barretos, com percepção dos entrevistados mais positivas, não se reconhece que os serviços de APS sejam suficientes para o atendimento a demandas e necessidades do conjunto da população. A situação se torna ainda mais grave em regiões como Manaus, cuja geografia e dispersão populacional criam dificuldades adicionais de acesso a serviços básicos de saúde, os quais precisam estar disponíveis em local que seja o mais próximo possível de onde as pessoas vivem e trabalham. Yamada (2011, apud Yin 17) destaca a relação entre tamanho do território, eficiência e acesso a serviços no campo da saúde, mostrando como resultado uma equação cruel: quanto menor a densidade populacional do município, mais difícil se torna prover a oferta, sendo maior o gasto per capita em saúde e pior o acesso aos serviços 17 .

A insuficiência percebida pelos entrevistados é ainda mais grave para a atenção especializada; nas regiões Cuiabana e Carbonífera foi consensual esta insuficiência (Figura 1). A oferta de SADT de média complexidade foi considerada insuficiente em todas as regiões, exceto em Barretos onde metade dos entrevistados a considerou suficiente. Em Barretos, assim como em grande parte do Estado de São Paulo, foram criados pelo governo estadual Ambulatórios Médico de Especialidades (AMEs) que ofertam consultas especializadas, procedimentos cirúrgicos ambulatoriais e SADT, todos geridos por organizações sociais, o que explica esse resultado.

A Figura 2 permite visualizar um dos maiores desafios estratégicos para a consolidação da APS no nosso país: a insuficiência de recursos humanos, especialmente médicos. A maior disponibilidade de enfermeiros para a APS é percebida em todas as regiões, sendo que os entrevistados referiram maior suficiência de dentistas na APS em PEBA e Barretos. A dificuldade de atração e fixação de profissionais, especialmente os médicos, gera escassez e má distribuição geográfica, reflexo da preferência destes profissionais em se fixar em regiões economicamente mais desenvolvidas e com maiores oportunidades profissionais 18 . No entanto, esse cenário pode ser revertido com políticas específicas e de desenvolvimento regional, como pode ser observado em PEBA, que se destaca frente as outras regiões. Uma das explicações plausíveis é a influência da Universidade do Vale do São Francisco, que ampliou a formação de recursos humanos para saúde na última década, e que conta com um movimento expressivo de formação e atuação de uma nova geração de médicos de família e comunidade no SUS.

Essa insuficiência se expressa na existência, em todas as regiões, de grupos populacionais sem cobertura de APS, especialmente nas regiões de Manaus, Cuiabana e PEBA. Os grupos citados são exatamente aqueles que apresentam maiores necessidades de atenção à saúde, dada suas inserções sociais, marcadas pelo viés de exclusão. Foram citadas as comunidades indígenas, rurais e ribeirinhas (Manaus), moradores de zonas do programa Minha Casa Minha Vida e regiões quilombolas (PEBA); comunidades ribeirinhas, área central de Cuiabá e zona rural (Cuiabana); assentamentos rurais 
Figura 1

Percepção dos entrevistados quanto à suficiência de cobertura da atenção primária à saúde (APS), de oferta de consultas especializadas e de oferta de Serviços de Apoio Diagnóstico e Terapêutico (SADT) de média complexidade. Regiões estudadas, 2015/2016.

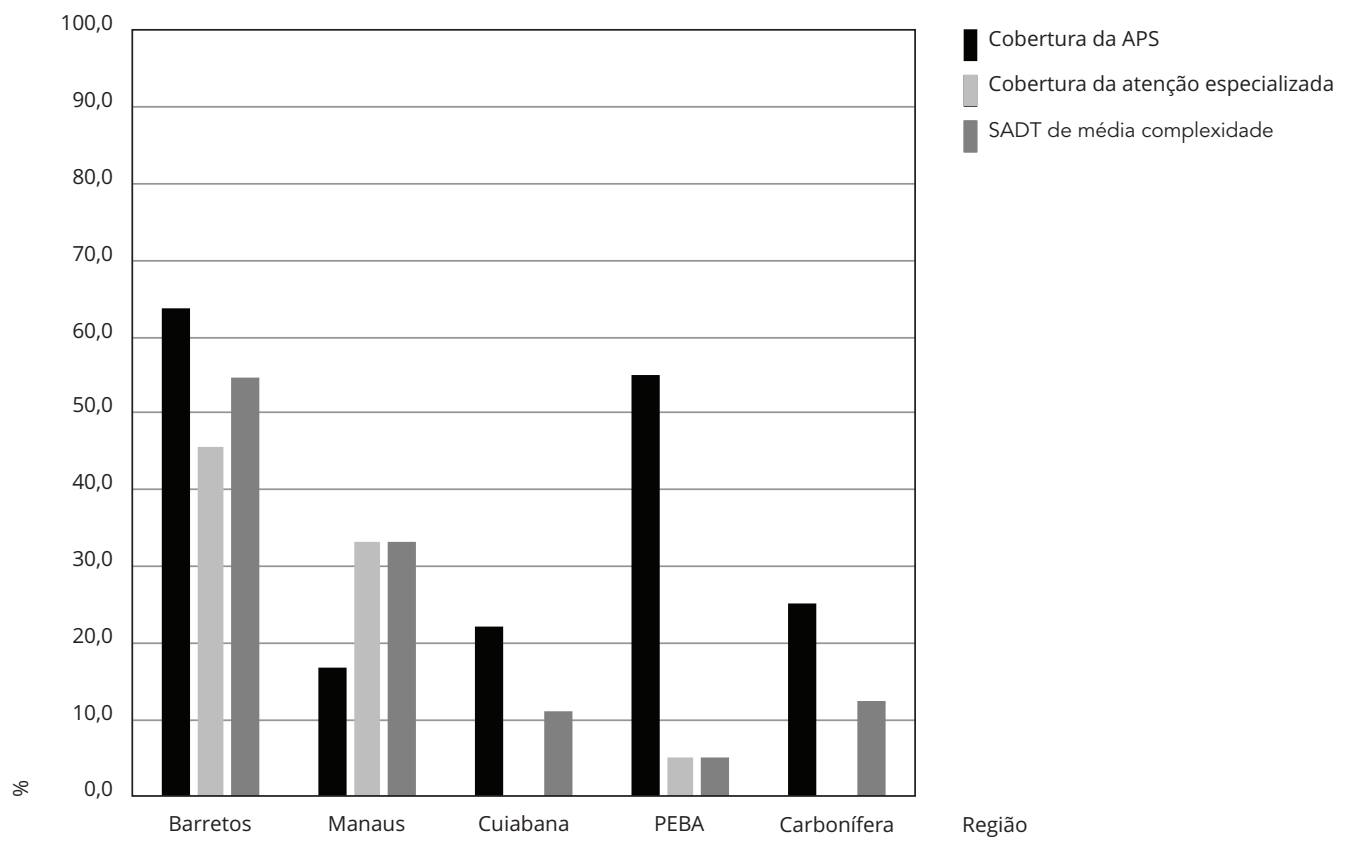

PEBA: Rede Interestadual de Atenção à Saúde do Vale Médio do São Francisco (Petrolina, Pernambuco e Juazeiro, Bahia).

(Barretos); e população indígena (Carbonífera). A existência de vazios assistenciais, manifestos pela falta de profissionais e serviços de saúde, pode ser considerada entrave ao avanço dos processos de regionalização. Na medida em que não houve aporte de novos recursos para a constituição das redes regionalizadas, o arranjo regional permaneceu condicionado aos recursos advindos do Programação Pactuada Integrada (PPI) e aos limites orçamentários dos municípios para financiar a atenção integral 3.

As regiões mais carentes e/ou remotas, com população em situação de extrema pobreza, são as que apresentam maior dificuldade de atenção à saúde em diversos cenários 9,19.

A utilização de critérios de risco e vulnerabilidade para a definição do número de pessoas sob a responsabilidade de uma UBS é percebida diferentemente por gestores e gerentes destas Unidades, em todas as regiões. Enquanto os gestores tendem a identificar a utilização de critérios de adscrição, os profissionais atuantes nos serviços de APS não os identificam no cotidiano dos serviços.

A natureza jurídica e o tipo de vínculo na contratação de recursos humanos nos serviços de APS, atenção especializada e SADT são distintos entre os estabelecimentos de APS e os demais. Nas cinco regiões os serviços de APS são praticamente constituídos por serviços próprios com recursos humanos com vínculos empregatícios como funcionários públicos estatutários ou contratados via Consolidação das Leis Trabalhistas (CLT). No entanto, a natureza jurídica é mais diversificada nos serviços que oferecem consultas especializadas e exames de SADT, com a presença de serviços privados contratados. A lógica de mercado desses últimos serviços, associada ao frequente monopólio, são extremamente difíceis de serem enfrentados pela maioria dos municípios brasileiros isoladamente, o que exige avanços na regionalização.

A presença de serviços contratualizados por Organizações Sociais de Saúde (OSS) para atenção especializada é observada somente em Barretos. Vale ressaltar que nesse município, no momento da pesquisa, discutia-se a proposta de passar a gestão das UBS do município polo para uma OSS, 


\section{Figura 2}

Percepção dos entrevistados quanto à suficiência de médicos, enfermeiros e dentistas na atenção primária à saúde (APS). Regiões estudadas, 2015/2016.

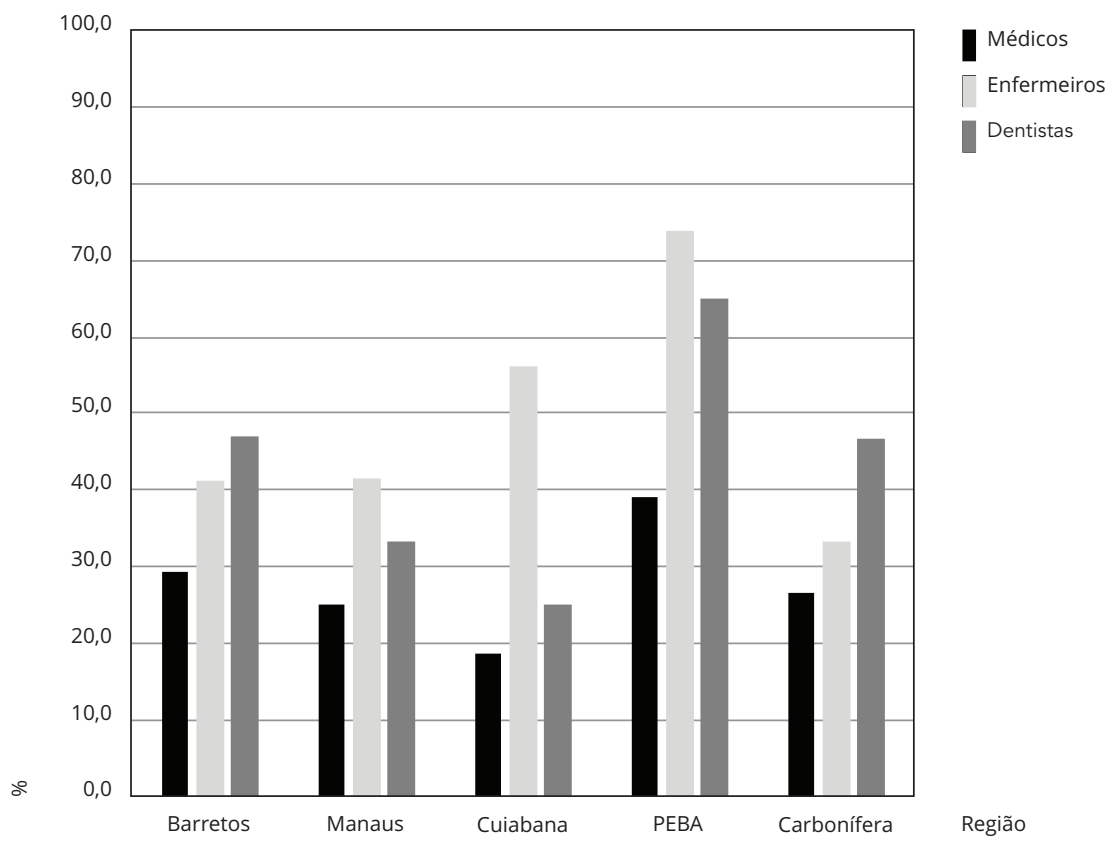

PEBA: Rede Interestadual de Atenção à Saúde do Vale Médio do São Francisco (Petrolina, Pernambuco e Juazeiro, Bahia).

seguindo a tendência da ampliação da contratualização de serviços de APS em curso no Estado de São Paulo 20.

Essas questões são ainda mais expressivas quando se reconhece que, para a integralidade da atenção, os serviços de APS necessitam da garantia do acesso a exames complementares e especialistas em tempo oportuno. Dado o perfil econômico e de porte populacional da maioria dos municípios brasileiros, é mandatório que os serviços especializados sejam oferecidos em escala regional.

Quanto ao investimento na APS, a maioria dos entrevistados considerou que houve aumento nos três anos anteriores, com exceção da região Carbonífera, na qual mais da metade dos entrevistados percebeu estagnação ou diminuição. Os resultados são coerentes com as diversas iniciativas de políticas do Ministério da Saúde para a melhoria da APS (Requalifica-UBS; Programa Mais Médicos, PMAQ) implantadas na última década 21.

Em todas as regiões, as esferas municipal e federal foram percebidas como as mais importantes para o financiamento na APS. Campos et al. 22 destacam que o modo indutivo de constituição da rede de atenção primária no Brasil, com a não definição da responsabilidade dos estados, possibilitou a redução da participação deste ente federado na elaboração, difusão e cofinanciamento da APS 22. Por outro lado, estudos sobre o custo da Estratégia Saúde da Família (ESF) corroboram que é o município que arca com grande parte do custeio 23,24. Na União Europeia, o maior protagonismo das esferas regionais e subnacionais foi correlacionado com melhor financiamento pelos níveis subnacionais 25 .

\section{Dimensão organização}

A organização dos serviços e ações de APS e sua integração aos outros níveis de atenção é ponto central para a garantia da integralidade do cuidado. Essa organização para ser efetiva necessita de planejamento, monitoramento e avaliação incorporados no cotidiano da gestão. 
Quando questionados sobre os instrumentos usados para o planejamento regional dos serviços, os pesquisados apresentaram respostas variadas entre as regiões, além de apontar instrumentos que não necessariamente atuam sobre o planejamento. Os exemplos mais citados foram o PMAQ-AB, Plano municipal de saúde e reuniões das próprias equipes de APS.

O Diagnóstico de Necessidades de Atenção à Saúde foi o instrumento mais referido, seguido pelo diagnóstico regional de capacidade instalada em todas as regiões. A percepção do uso desses instrumentos foi menor nas regiões de Manaus e Carbonífera. Não obstante, 20\% dos entrevistados não sabiam responder à questão.

Há referência da avaliação por uso de indicadores de desempenho dos serviços da APS nas regiões, com exceção da região Carbonífera. Nessa última, menos de um terço dos respondentes identificou a sua existência. No entanto, quando questionados sobre quais seriam esses indicadores, as respostas nas outras regiões continuaram dispersas, incluindo o PMAQ-AB e o Sispacto (pactuação interfederativa de indicadores). Novamente, com exceção da região Carbonífera, foram citados os gestores municipais de saúde e os coordenadores municipais de APS como os principais responsáveis pelo processo de monitoramento e avaliação.

O PMAQ-AB, iniciado em 2011, objetivou a ampliação de acesso e qualidade da APS por meio de indução. Atingiu a maioria das UBS brasileiras, sendo o principal instrumento de avaliação referido pelos entrevistados. No entanto, profissionais não possuem o preparo e o domínio adequados para realizar o monitoramento e a análise dos indicadores 26 , o que pode refletir em prejuízo no processo contínuo de planejamento das ações.

A coordenação do cuidado pela APS deve ser entendida como uma articulação harmoniosa entre diferentes profissionais e serviços de saúde, que tem por objetivo comum garantir uma atenção integral e de qualidade aos usuários, de acordo com as suas necessidades, combatendo a fragmentação dos sistemas de saúde 27.

Em relação à porta de entrada preferencial para a RAS, os gerentes de UBS são quase unânimes em afirmar que esta é a UBS. A exceção é Manaus onde se observa variação nas repostas. Por outro lado, quando questionados sobre a contribuição da APS na resolutividade dos problemas de saúde, em média 52,5\% afirmam contribuição positiva, sendo que em Manaus e Cuiabana este percentual cai para 33\%.

A gestão da APS nas regiões tem contribuído para a melhoria do fluxo assistencial e para a integração dos serviços da rede, segundos os entrevistados. Essas percepções são maiores em Barretos e menores em Manaus. Mais de dois terços dos respondentes evidenciaram a existência de integração da APS com os demais serviços de saúde nas regiões de PEBA, Barretos e Carbonífera. Diferentemente da situação das regiões de Manaus e Cuiabana, onde apenas um terço identificou integração.

A avaliação sobre agendamento em tempo oportuno de algumas consultas e exames em especialidades pode ser visualizada na Figura 3, que apresenta o escore médio aferido. A atenção oportuna implica a prestação do serviço necessário em tempo adequado, de modo a se evitar complicações, o que é crucial na evolução das doenças crônicas. Do ponto de vista dos recursos em saúde, isso significa uso racional e eficiente, com alocação apropriada, o que reduz gastos desnecessários do sistema. O escore, que varia entre 1 e 5 , é pior para exames de fundo de olho (escore médio $=2,68$ ), e entre as regiões a que apresenta o pior índice é Barretos (escore médio = 2,37), seguida por Manaus $(2,49$ ), PEBA (2,95), Cuiabana $(2,99)$ e Carbonífera $(3,19)$. A oferta em tempo inoportuno contribui para dificuldades na experiência de uso dos serviços de APS, e alguns estudos indicam que estes são pouco reconhecidos pelos usuários como lugar de cuidado regular e porta de entrada para acessar os demais recursos em saúde. Ainda prevalece a visão da APS como locus de busca e acesso a procedimentos simples, como medicamentos ou aferição da pressão arterial. Esse perfil é encontrado, inclusive, entre usuários frequentes dos serviços 28,29 .

A Tabela 3 apresenta os escores obtidos nas questões relacionadas à integração entre equipes e serviços (APS-atenção especializada); acompanhamento pela APS das situações de maior risco em outros pontos de atenção. $\mathrm{O}$ acesso à atenção especializada, via o encaminhamento pela APS, ainda é muito frágil nas regiões de Manaus, Cuiabana e Carbonífera, que são exatamente aquelas com a menor percepção de suficiência da atenção especializada, demonstrando que em situações de maior precariedade se torna mais difícil a organização dos fluxos, levando ao que pode ser chamado de uma potencialização adversa, negativa. Quando o usuário necessita de consulta de atenção especializada, os percursos mais citados foram: o agendamento pela UBS, que informa posteriormente ao usuário, 
Figura 3

Percepção dos entrevistados quanto à realização em tempo oportuno de consultas especializadas e exames complementares selecionados. Regiões estudadas, 2015/2016.

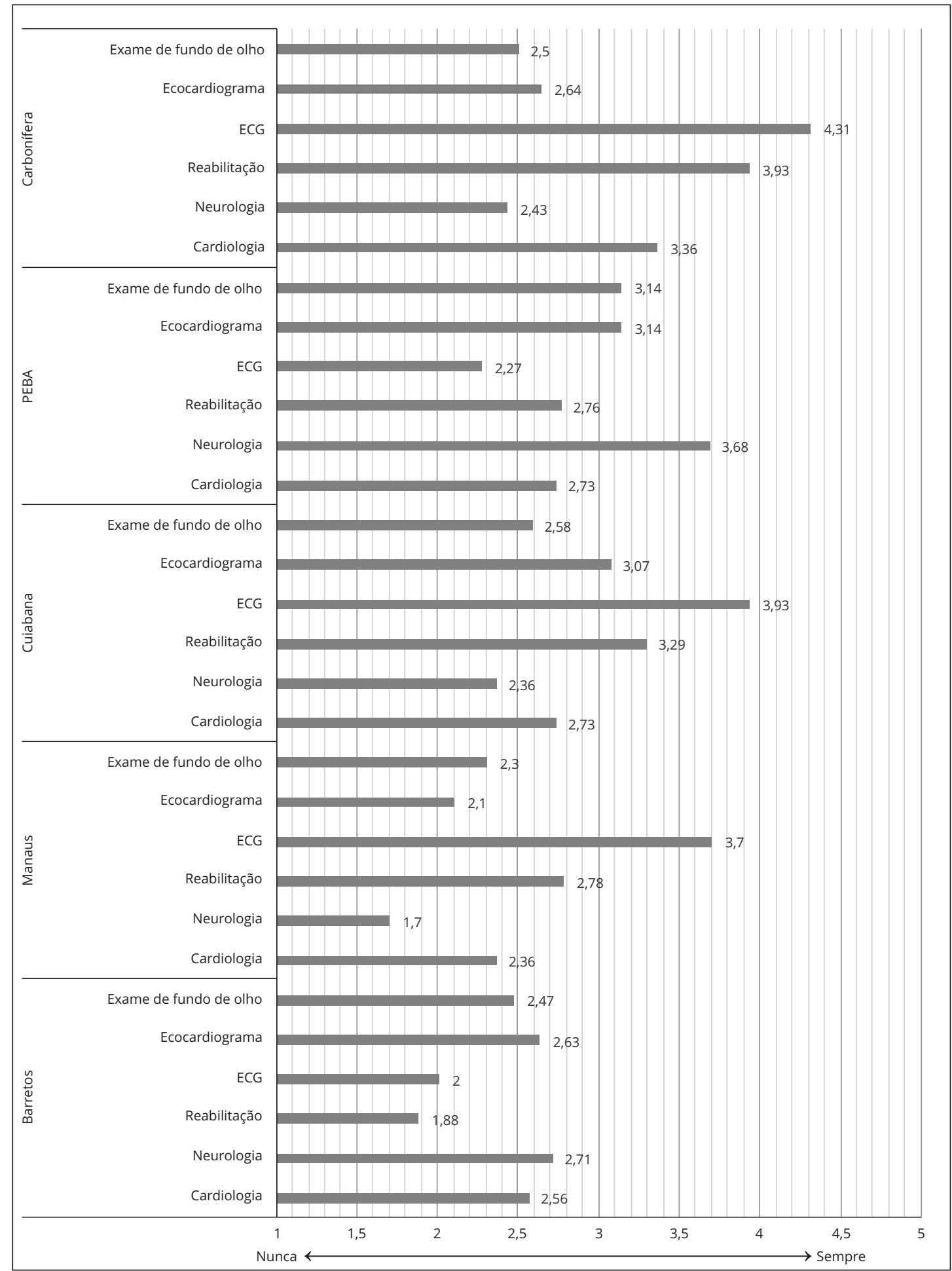

ECG: eletrocardiograma; PEBA: Rede Interestadual de Atenção à Saúde do Vale Médio do São Francisco (Petrolina, Pernambuco e Juazeiro, Bahia). 


\section{Tabela 3}

Percepção dos entrevistados quanto à Integração entre a atenção primária à saúde (APS) e a atenção especializada. Regiões estudadas, $2015 / 2016$.

\begin{tabular}{|c|c|c|c|c|c|}
\hline & $\begin{array}{l}\text { Barretos } \\
\text { Escore }\end{array}$ & $\begin{array}{c}\text { Manaus } \\
\text { Escore }\end{array}$ & $\begin{array}{c}\text { Cuiabana } \\
\text { Escore }\end{array}$ & $\begin{array}{l}\text { PEBA } \\
\text { Escore }\end{array}$ & $\begin{array}{l}\text { Carbonífera } \\
\text { Escore }\end{array}$ \\
\hline UBS é notificada quando o usuário é atendido em serviços de urgência e emergência & 1,60 & 1,50 & 1,25 & 4,00 & 1,25 \\
\hline UBS é notificada quando o usuário é atendido em serviços hospitalares & 1,80 & 1,50 & 1,25 & 4,00 & 1,25 \\
\hline Após alta hospitalar a UBS recebe relatório de internação & 1,50 & 1,50 & 1,80 & 4,00 & 1,00 \\
\hline Se informado da alta hospitalar, ACS faz visita domiciliar ao usuário & 4,20 & 4,50 & 4,25 & 2,14 & 3,33 \\
\hline $\begin{array}{l}\text { Se informado da alta hospitalar, o médico ou o enfermeiro da unidade de APS faz } \\
\text { visita domiciliar ao usuário }\end{array}$ & 3,30 & 4,00 & 3,25 & 2,00 & 2,25 \\
\hline APS recebe contrarreferência escrita da atenção especializada & 1,90 & 1,80 & 2,11 & 3,29 & 2,22 \\
\hline Acesso à atenção especializada depende de encaminhamento pela APS & 4,50 & 3,83 & 4,11 & 1,64 & 4,89 \\
\hline Ocorre atendimento pela atenção especializada sem encaminhamento pela APS & 1,90 & 2,60 & 2,78 & 3,62 & 1,44 \\
\hline
\end{tabular}

ACS: agentes comunitários de saúde; PEBA: Rede Interestadual de Atenção à Saúde do Vale Médio do São Francisco (Petrolina, Pernambuco e Juazeiro, Bahia); UBS: unidades básicas de saúde.

Nota: Escore médio variando entre 1 e 5.

e o agendamento em central de marcação em todas as regiões, com exceção da Cuiabana onde não há referência à central de marcação.

Os resultados deste estudo são semelhantes aos de Chaves et al. 30 que, ao analisarem a integração das equipes de APS com a atenção especializada, identificaram apenas 13\% destas em cenário de alta integração. Garantir a comunicação entre especialistas e a APS tem sido considerado um ponto central no combate à fragmentação, melhoria da atenção e diminuição de custos em diversos cenários, inclusive com o desenho de propostas que fogem ao desenho de equipamentos de atenção especializada e APS estanques 31 . Embora a APS seja considerada a principal porta de entrada do sistema, ainda falta muito para que se caracterize como ordenadora da rede nas regiões analisadas. A escala regional é central para a reversão desse quadro e, também, para a própria qualificação da atenção especializada.

Ampliar o escopo da APS, no sentido de torná-la mais efetiva/resolutiva, também é essencial para a garantia de cuidados coordenados, contínuos, integrados e integrais. Experiências relacionadas ao fortalecimento do trabalho em equipe, ampliação e valorização das ações clínicas do enfermeiro e do Núcleo de Apoio à Saúde da Família (NASF), da cobertura por equipes de saúde bucal, ampliação do leque de serviços e adensamento do arsenal tecnológico das UBS, por exemplo, poderiam contribuir para que a população reconhecesse a atenção básica como local de prestação de cuidados resolutivos e de qualidade 32 .

No que diz respeito à dimensão organização, os resultados do presente estudo assinalam as visíveis dificuldades para se romper a fragmentação dos serviços e a ausência de um modus operandi capaz de atender às necessidades dos usuários e às especificidades de cada território regional. As heterogeneidades observadas intrarregionalmente e entre regiões revelam diferenças que podem estar relacionadas a diferentes fatores como desenvolvimento tecnológico, aprendizado institucional prévio na trajetória de organização do sistema de saúde, background cultural e institucional, entre outros. Isso impõe um grande desafio, posto que os problemas e soluções exigem acurácia de investigação empírica na análise de cada situação particular, dado que, como bem assinala Viana et al. 33, não são soluções normativas ou burocráticas que darão conta de resolver de forma eficiente e eficaz os obstáculos que se colocam para a integração e coordenação do cuidado de qualidade em contextos regionais diversos. Nesse particular, cabe ressaltar a multiplicidade de atores, expertises, saberes e experiências que precisam ser mediados por meio de mecanismos de ausculta e compartilhamento de poder, tendo em vista a organização de fluxos e o compartilhamento de responsabilidades que permitem dar concretude à operação e funcionamento autorregulatório das redes nas regiões de saúde. 


\section{Considerações finais}

A discussão sobre o fortalecimento da APS e a construção da RAS tratam do fortalecimento do próprio SUS como sistema público, universal, equânime e integral. Nas regiões estudadas foram observadas importantes fragilidades, em diferentes graus, nas dimensões da política, estrutura e organização. As fragilidades não são pequenas, e certamente ocorrem em outras RAS. A APS ainda não é uma porta de entrada efetiva porta e preferencial no sistema de saúde nos cenários estudados. As condições estruturais dos serviços de APS são limitadas, há baixa capacidade de resolução clínica e de incorporação de instrumentos e mecanismos de coordenação do cuidado. Pode-se afirmar que os diversos esforços empreendidos na formulação de políticas de fortalecimento da APS não foram suficientes para criar as condições necessárias à sua atuação no espaço regional.

A fragilidade das estruturas administrativas descentralizadas em nível regional também contribui para esse cenário. Essas instâncias precisam ampliar enormemente seu poder político e capacidade técnica, tornando-se capazes de exercer o seu papel no planejamento da RAS, formulando estratégias de financiamento e da garantia de cuidados de saúde com o desenvolvimento de mecanismos de coordenação assistencial.

Superar as inúmeras fragilidades é imperativo para que uma APS abrangente e articulada se torne uma realidade. Deve-se ter claro que o desafio brasileiro para a construção de uma APS forte e abrangente é parte do desafio maior de construção de RAS, em um contexto de autonomia da gestão municipal, articulada em âmbito regional, com protagonismo da esfera estadual, em conformidade com os princípios do SUS.

\section{Colaboradores}

A. Bousquat e P. H. S. Mota contribuíram no desenho do estudo, análise e interpretação dos dados, escrita, revisão e aprovação final do manuscrito. L. Giovanella contribuiu na interpretação dos dados, escrita, revisão e aprovação final do manuscrito. M. C. R. Fausto, M. G. Medina, C. L. Martins, P. F. Almeida e E. M. S. Campos contribuíram na escrita, revisão e aprovação final do manuscrito.

\section{Informações adicionais}

ORCID: Aylene Bousquat (0000-0003-2701-1570); Lígia Giovanella (0000-0002-6522-545X); Marcia Cristina Rodrigues Fausto (0000-0003-0746-3684); Maria Guadalupe Medina (0000-0001-7283-2947); Cleide Lavieri Martins (0000-0001-7688-0516); Patty Fidelis Almeida (0000-0003-1676-3574); Estela Marcia Saraiva Campos (0000-0002-35699785); Paulo Henrique dos Santos Mota (00000003-3507-3958).

\section{Agradecimentos}

Projeto Região e redes (Chamada MCTI/CNPq/ CT-Saúde/MS/SCTIE/Decit no 41/201). 


\section{Referências}

1. Kuschnir R, Chorny AH. Redes de atenção à saúde: contextualizando o debate. Ciênc Saúde Colet 2010; 15:2307-16.

2. Kuschnir R. Organizando redes de atenção à saúde: perfis assistenciais, articulação entre níveis e organização de linhas de cuidado. In: Kuschnir R, Fausto MCR, editores. Gestão de redes de atenção à saúde. Rio de Janeiro: Educação à Distância, Escola Nacional de Saúde Pública Sergio Arouca, Fundação Oswaldo Cruz; 2014. p. 129-68.

3. Almeida PF, Santos AM, Santos VP, Silveira Filho RM. Integração assistencial em região de saúde: paradoxo entre necessidades regionais e interesses locais. Saúde Soc 2016; 25:320-35.

4. Vargas I, Mogollon-Perez AS, Unger J-P, daSilva MRF, De Paepe P, Vazquez M-L. Regional-based Integrated Healthcare Network policy in Brazil: from formulation to practice. Health Policy Plan 2015; 30:705-17.

5. Lima LD, Viana ALA, Machado CV, Albuquerque MV, Oliveira RG, Iozzi FL, et al. Regionalização e acesso à saúde nos estados brasileiros: condicionantes históricos e político-institucionais. Ciênc Saúde Colet 2012; 17:2881-92.

6. Moreira LMC, Ferré F, Andrade EIG. Financiamento, descentralização e regionalização: transferências federais e as redes de atenção em Minas Gerais, Brasil. Ciênc Saúde Colet 2017; 22:1245-56.

7. Albuquerque MV, Viana ALA, Lima LD, Ferreira MP, Fusaro ER, Iozzi FL, et al. Desigualdades regionais na saúde: mudanças observadas no Brasil de 2000 a 2016. Ciênc Saúde Colet 2017; 22:1055-64.

8. Spedo SM, Pinto NRS, Tanaka OY. A regionalização intramunicipal do Sistema Único de Saúde (SUS): um estudo de caso do Município de São Paulo - SP, Brasil. Saúde Soc 2010; 19:533-46.

9. Travassos C, Oliveira EXG, Viacava F. Desigualdades geográficas e sociais no acesso aos serviços de saúde no Brasil: 1998 e 2003. Ciênc Saúde Colet 2006; 11:975-86.

10. Fausto MCR, Giovanella L, Mendonça MHM, Fonseca HMS, Lima JG. A posição da Estratégia Saúde da Família na rede de atenção à saúde na perspectiva das equipes e usuários participantes do PMAQ-AB 2012. Saúde Debate 2014; 38(N spec):13-33.

11. Macinko J, Dourado I, Aquino R, Bonolo PF, Lima-Costa MF, Medina MG, et al. Major expansion of primary care in Brazil linked to decline in unnecessary hospitalization. Health Aff (Millwood) 2010; 29:2149-60.

12. Hone T, Rasella D, Barreto M, Atun R, Majeed A, Millett C. Large reductions in amenable mortality associated with Brazil's primary care expansion and strong health governance. Health Aff (Millwood) 2017; 36:149-58.
13. Viana ALA, Ferreira MP, Cutrim MA, Fusaro ER, Souza MR, Mourão L, et al. The regionalization process in Brazil: influence on policy, structure and organization dimensions. Rev Bras Saúde Matern Infant 2017; 17 Suppl 1:S27-43.

14. Viana ALA, Bousquat A, Ferreira MP, Cutrim MAB, Uchimura LYT, Fusaro ER, et al Region and networks: multidimensional and multilevel approaches to analyze the health regionalization process in Brazil. Rev Bras Saúde Matern Infant 2017; 17 Suppl 1:S7-16.

15. Bousquat A, Giovanella L, Fausto MCR, Fusaro ER, Mendonça MHM, Gagno J, et al. Tipologia da estrutura das unidades básicas de saúde brasileiras: os 5 R. Cad Saúde Pública 2017; 33:e0037316.

16. Abrucio FL, Couto CG. A redefinição do papel do Estado no âmbito local. São Paulo Perspect 1996; 10:40-7.

17. Yin JN. Economias de escala e eficiência de gastos na saúde: novas evidências [Dissertação de Mestrado]. São Paulo: Fundação Getúlio Vargas; 2011.

18. Van Stralen ACS, Massote AW, Carvalho CL, Girardi SN. Percepção de médicos sobre fatores de atração e fixação em áreas remotas e desassistidas: rotas da escassez. Physis (Rio J.) 2017; 27:14-72.

19. Cavalcante IMS. Acesso e acessibilidade aos serviços de saúde em três quilombos da Amazônia Paraense: um olhar antropológico [Dissertação de Mestrado]. Belém: Universidade Federal do Pará; 2011.

20. Greve J, Schattan V. Evaluating the impact of contracting out basic health care services in the state of São Paulo, Brazil. Health Policy Plan 2017; 32:923-33.

21. Giovanella L, Mendonça MHM, Fausto MCR, Almeida PF, Bousquat A, Lima JG, et al. A provisão emergencial de médicos pelo Programa Mais Médicos e a qualidade da estrutura das unidades básicas de saúde. Ciênc Saúde Colet 2016; 21:2697-708.

22. Campos GWS, Pereira Júnior N. A atenção primária e o programa Mais Médicos do Sistema Único de Saúde: conquistas e limites. Ciênc Saúde Colet 2016; 21:2655-63.

23. Castro ALB, Machado CV. A política de atenção primária à saúde no Brasil: notas sobre a regulação e o financiamento federal. Cad Saúde Pública 2010; 26:693-705.

24. Mendes A, Marques RM. O financiamento da atenção básica e da Estratégia Saúde da Família no Sistema Único de Saúde. Saúde Debate 2014; 38:900-16.

25. European Union. The management of health systems in the EU Member States - the role of local and regional authorities. Luxembourg: Publications Office of the Europe Union; 2012. 
26. Shimizu HE, Fragelli TBO. Competências profissionais essenciais para o trabalho no Núcleo de Apoio à Saúde da Família. Rev Bras Educ Méd 2016; 40:216-25

27. Terraza Núñez R, Vargas Lorenzo I, Vázquez Navarrete ML. La coordinación entre niveles asistenciales: una sistematización de sus instrumentos y medidas. Gac Sanit 2006; 20:48595.

28. Bousquat A, Giovanella L, Campos EMS, Almeida PF, Martins CL, Mota PHS, et al. Atenção primária à saúde e coordenação do cuidado nas regiões de saúde: perspectiva de gestores e usuários. Ciênc Saúde Colet 2017; 22:1141-54.

29. Cecilio LCO, Andreazza R, Carapinheiro G, Araújo EC, Oliveira LA, Andrade MGG, et al. A atenção básica à saúde e a construção das redes temáticas de saúde: qual pode ser o seu papel? Ciênc Saúde Colet 2012; 17:2893-902.
30. Chaves LA, Jorge AO, Cherchiglia ML, Reis IA, Santos MAC, Santos AF, et al. Integração da atenção básica à rede assistencial: análise de componentes da avaliação externa do PMAQ-AB. Cad Saúde Pública 2018; 34:e00201515.

31. Biagetti B, Aulinas A, Dalama B, Nogués R, Zafón C, Mesa J. Communication between primary care and physician specialist: Is it improving? Rev Calid Asist 2015; 30:175-81.

32. Almeida PF, Santos AM. Primary Health Care: care coordinator in regionalized networks? Rev Saúde Pública 2016; 50:80.

33. Viana ALA, Bousquat A, Melo GA, Negri Filho A, Medina MG. Regionalização e redes de saúde. Ciênc Saúde Colet 2018; 23:1791. 


\section{Abstract}

Primary health care (PHC), conceived as the coordinator of care and the backbone of the regionalized health care network, is one of the conditioning factors in health. The article aims to identify the political, structural, and organizational dimensions of PHC in different regions of Brazil and assumes that these dimensions can at least partially explain the functioning of $\mathrm{PHC}$ in the regional scenario. A total of 84 interviews were held with key actors in five health regions. The regions were selected on the basis of the diversity of socioeconomic, territorial, and organizational situations in the health system. Despite the heterogeneity in the regionalized health care networks, some common traits were seen. The political dimension revealed fragilities in intergovernmental cooperation and state-level leadership, in addition to the inability of the Bipartite Inter-Managers Commission to serve as a space for planning and negotiated agreements. The structural dimension showed a clear insufficiency of conditions to minimally ensure the execution of essential functions in PHC. Critical points are the shortage, poor distribution, and deficient qualifications of human resources and underfunding of activities. The organizational dimension reveals difficulties in breaking with the fragmentation of services. PHC is unable to fulfill its role as coordinator of care, and there is lack of a modus operandi capable of meeting users' needs, considering each region's specificities. Overcoming the above-mentioned constraints is essential for strengthening the Brazilian Unified National Health System as a public, universal, equitable, and comprehensive system.

Primary Health Care; Regional Health Planning; Health Systems

\section{Resumen}

La atención primaria de salud (APS), concebida como coordinadora del cuidado y organizadora de la red regionalizada de atención de salud (RAS), es uno de los condicionantes de la dinámica regional de salud. El objetivo del artículo es identificar las dimensiones: política, de estructura y de organización de la APS en diferentes regiones de Brasil; se asume que estas dimensiones pueden explicar, si no todo, por lo menos una parte importante del funcionamiento de la APS en el escenario regional. Se realizaron 84 entrevistas con actores clave en cinco regiones de salud. Las regiones se seleccionaron basándose en la diversidad de situaciones socioeconómicas, territoriales y de organización del sistema de salud. A pesar de la heterogeneidad de las RAS se notan rasgos comunes. En la dimensión política se advirtió la fragilidad en la cooperación intergubernamental y en el protagonismo de la esfera estatal, además de la incapacidad de la Comisión Intergestores Bipartita para convertirse en un espacio de planificación y pactos. En la dimensión estructura quedó patente la insuficiencia de las condiciones que aseguren minimamente la ejecución de las funciones esenciales de la APS. Puntos críticos son la escasez, mala distribución y problemas de cualificación de recursos humanos, además de la escasa financiación de las acciones. En la dimensión organización son visibles las dificultades para romper la fragmentación de los servicios. La APS no consigue asumir su papel de coordinadora del cuidado, y se observa la ausencia de un modus operandi capaz de atender a las necesidades de los usuarios, considerando las especificidades de cada región. La superación de los obstáculos identificados es primordial para el fortalecimiento del propio Sistema Único de Salud como sistema público, universal, ecuánime e integral.

Atención Primaria de Salud; Regionalización; Sistemas de Salud
Recebido em 22/Mai/2018

Versão final reapresentada em 29/Nov/2018

Aprovado em 11/Dez/2018 